\title{
Manifestações de sintomas somáticos em pacientes com Fibromialgia: uma revisão narrativa
}

\author{
Manifestations of somatic symptoms in patients with Fibromyalgia: a narrative review \\ Manifestaciones de síntomas somáticos en pacientes con Fibromialgia: una revisión \\ narrativa
}

Lael Andrade Menezes Filho ${ }^{1 *}$, Arlys Emanuel Mendes da Silva Santos ${ }^{2}$, Paula Medina Cribari ${ }^{3}$, Luiz Valério Costa Vasconcelos ${ }^{4}$, Juliana Carvalho Rezende3, Ana Leticia Lira Paulino ${ }^{5}$, Hallana de Lucena Araujo ${ }^{4}$, Gabrielle Hespanha Franco ${ }^{6}$, Joana Bader Sadala Brandão ${ }^{7}$, Leonardo Soares Prates ${ }^{8}$.

\section{RESUMO}

Objetivo: Expor os sintomas somáticos de Fibromialgia, analisar suas repercussões na qualidade de vida dos portadores desta patologia e estudar intervenções que minimizem essa problemática. Revisão bibliográfica: A Fibromialgia é uma síndrome de dor crônica associada a sintomas somáticos e cognitivos, fadiga e alterações no sono, que afetam diretamente a vida dos pacientes devido ao conjunto sintomatológico relacionado às dores. No Brasil há a prevalência de $2 \%$ de Fibromialgia, com proporção de 1 homem a cada 5,5 mulheres e, em relação à epidemiologia dos sintomas somáticos, de um total de 140 pacientes com esta patologia, 91 tinham pelo menos um sintoma somático. Sua etiopatogenia é complexa, multifacetada, não conhecida por completo, e envolve fatores extrínsecos e intrínsecos. Para critérios diagnósticos, atualmente utiliza-se o Índice de Dor Generalizada (IDG), Escala de Gravidade dos Sintomas (EGS) e a presença ou história de sintomas somáticos. Atualmente são utilizados diversos tipos de tratamentos farmacológicos e não farmacológicos. Considerações finais: $\mathrm{O}$ trabalho elucida que há uma prevalência de sintomas somáticos nos pacientes diagnosticados com Fibromialgia, porém é necessário que seja desenvolvido meios de mensuração da dor dos pacientes de forma mais específica, assim como aumentar estratégias que proporcionam uma intervenção precoce.

Palavras-chave: Fibromialgia, Sintomas somáticos, Transtornos somatoformes, Prevalência.

\begin{abstract}
Objective: Expose the somatic symptoms of Fibromyalgia, analyze their impact on the quality of life of patients with this pathology and study interventions to minimize this problem. Bibliographic review: Fibromyalgia is a syndrome of chronic pain associated with somatic and cognitive symptoms, fatigue and sleep disorders that directly affect the lives of patients due to the symptomatology related to joint pain. In Brazil there is a prevalence of $2 \%$ of Fibromyalgia, with a proportion of 1 man for every 5.5 women and, in relation to the epidemiology of somatic symptoms, of a total of 140 patients with this pathology, 91 had at least one somatic symptom. Its etiopathogenesis is complex, multifaceted, not completely known, and involves extrinsic and intrinsic factors. For diagnostic criteria, the widespread pain index (WPI), Symptom Severity Scale (SSS) and the presence or history of somatic symptoms are currently used. Currently, several types of pharmacological and nonpharmacological treatments are used. Final considerations: The work elucidates that there is a prevalence of somatic symptoms in patients diagnosed with Fibromyalgia, however it is necessary to develop means of measuring patients' pain in a more specific way, as well as to increase strategies that provide an early intervention.
\end{abstract}

Keywords: Fibromyalgia, Somatic symptoms, Somatoform disorders, Prevalence.

\footnotetext{
1 Universidade Federal da Bahia (UFBA), Vitória da Conquista - BA. *E-mail: lael.filho@ufba.br

2 Universidade Federal do Piauí (UFPI), Teresina - PI.

${ }^{3}$ Centro Universitário FAMINAS (UNIFAMINAS), Muriaé - MG.

4 Universidade de Fortaleza (UNIFOR), Fortaleza - CE.

5 Unichristus (UC), Fortaleza - CE.

${ }^{6}$ Faculdade Ciências Médicas de Minas Gerais (FCMMG), Belo Horizonte - MG.

7 Universidade Nilton Lins (UNL), Manaus - AM.

8 Universidade Federal de Juiz de Fora (UFJF), Juiz de Fora - MG.
} 
RESUMEN

Objetivo: Exponer los síntomas somáticos de la Fibromialgia, analizar sus repercusiones en la calidad de vida de los pacientes con esta patología y estudiar intervenciones que minimicen este problema. Revisión bibliográfica: La fibromialgia es un síndrome de dolor crónico asociado con síntomas somáticos y cognitivos, fatiga y cambios en el sueño, que afectan directamente la vida de los pacientes debido a la serie de síntomas relacionados con el dolor. En Brasil existe una prevalencia del 2\% de Fibromialgia, con una proporción de 1 hombre por cada 5,5 mujeres y, en relación a la epidemiología de los síntomas somáticos, de un total de 140 pacientes con esta patología, 91 tenían al menos un síntoma somático. Su etiopatogenia es compleja, multifacética, no completamente conocida e involucra factores extrínsecos e intrínsecos. Para los criterios de diagnóstico, actualmente se utilizan el Índice de Dolor Generalizado (GDI), la Escala de Gravedad de los Síntomas (EGS) y la presencia o antecedentes de síntomas somáticos. Actualmente se utilizan varios tipos de tratamientos farmacológicos y no farmacológicos. Consideraciones finales: El trabajo dilucida que existe una prevalencia de síntomas somáticos en pacientes diagnosticados de Fibromialgia, sin embargo es necesario desarrollar medios para medir el dolor de los pacientes de manera más específica, así como incrementar estrategias que brinden una intervención temprana.

Palabras clave: Fibromialgia, Síntomas somáticos, Trastornos somatomorfos, Prevalencia.

\section{INTRODUÇÃO}

A Fibromialgia (FM) é um distúrbio polissintomático caracterizado principalmente por dor musculoesquelética crônica e generalizada, contudo, outros sintomas como fadiga, depressão, distúrbios do sono, sensibilidades sensoriais, comprometimento cognitivo, problemas digestivos, entre outros também estão associados. Por consequência, muitas pessoas que convivem com esse distúrbio referem sofrimento específico da dor e sofrimento psicológico associado, sendo assim uma condição incapacitante, interferindo em vários domínios funcionais, incluindo saúde física, social e mental e impactando a qualidade de vida (HÄUSER W, et al., 2015; BIRTANE M, et al., 2007; HADLANDSMYTH K, et al., 2017).

A FM apresenta-se como a terceira patologia musculoesquelética mais frequente no que concerne à prevalência, atrás apenas da dor lombar e osteoartrite. A prevalência é equivalente à idade dos indivíduos, tendo seu ápice na faixa etária de 35 a 60 anos. Afeta cerca de $2-5 \%$ da população mundial, sendo mais prevalente no sexo feminino, em uma proporção de 8-9: 1-2. Estima-se que a prevalência de FM no Brasil seja em torno de 2,5-4\%, apresentando uma proporção de 1 homem para casa 5,5 mulheres. Apesar de ser uma patologia heterogênea comumente encontrada na população geral, a FM permanece sem uma etiologia evidentemente definida, consenso clínico da doença ou tratamentos eficazes (SOUZA JB e PERISSINOTTI DMN, 2018).

A dificuldade para obter informações concretas sobre a fisiopatologia da FM é consequência da versatilidade do quadro clínico entre os pacientes, a complexidade para fechar o diagnóstico e diferenças entre as necessidades dos indivíduos. Até o momento, a teoria fisiopatológica mais adotada é a teoria da centralização: ocorre um aumento da sensibilidade central acarretando em uma resposta acentuada ao estímulo experimental de dor, juntamente com mudanças na estrutura e função de neurotransmissores centrais envolvidos na ação inibitória da dor (BHARGAVA J e HURLEY JA, 2020).

Dor e doença mental compartilham uma relação muito próxima. No Transtorno de Sintomas Somáticos, os sintomas podem ou não estar relacionados a uma condição física, mas a reação psicológica é sempre significativamente presente. Dessa forma, o Transtorno de Sintomas Somáticos gera níveis exacerbados e exagerados de sofrimento, onde o quadro clínico pode se restringir a um órgão ou envolver um ou mais órgãos e sistemas corporais diferentes. Apesar dos avanços no entendimento sobre os mecanismos da dor, os pacientes com dor crônica ainda são tratados como um grupo homogêneo, frequentemente havendo fracasso no tratamento (VLAEYEN JWS e LINTON SJ, 2012; MCBETH J, et al., 2001; BRAUN A, et al., 2020).

O manejo do Transtorno de Sintomas Somáticos é agravado pela falta de consenso sobre seus critérios diagnósticos, principalmente pela ausência de distúrbios identificáveis, e pela resposta ineficaz às estratégias terapêuticas infundadas. Esses desafios contribuem para o surgimento de suspeitas sobre a validade dos 
sintomas do Transtorno de Sintomas Somáticos e descrença sobre os mesmos, sobretudo pela hipótese de que esses sintomas constituem questões unicamente psicológicas, assim, não estando relacionadas a questões físicas. Ou seja, em geral, entre profissionais e pacientes, persistem dúvidas se essas manifestações clínicas resultam de doenças com fisiopatologia identificável e tratável ou de doenças psicossomáticas. No entanto, apesar de haver claramente uma relação com experiências ambientais externas (por exemplo, trauma, estilo de vida socioeconômico ou fatores relacionados ao estresse), tais suspeitas são refutadas pelo início abrupto e severidade de muitos distúrbios com sintomas somáticos, características presentes mesmo entre indivíduos previamente saudáveis física e mentalmente (ALBRECHT PJ e RICE FL, 2016).

Diante do exposto, o Transtorno de Sintomas Somáticos parece ser relativamente comum em fibromialgia, constituindo um alvo potencial de intervenções para estes pacientes frequentemente negligenciados. Assim, o objetivo desse estudo foi conhecer a prevalência de sintomas somáticos e suas repercussões na qualidade de vida entre indivíduos com fibromialgia, assim como buscar maneiras de intervenções para minimizar essa problemática.

\section{REVISÃO BIBLIOGRÁFICA}

\section{Fisiopatologia}

A FM é compreendida como uma síndrome de dor crônica associada a sintomas somáticos e cognitivos, fadiga e alterações no sono, sendo sua etiopatogenia complexa, multifacetada e não conhecida por completo (MARQUES AP, et al., 2017). A Organização Mundial da Saúde reconheceu a FM com o código do CID-10 M79.7 (HARRINSON J, et al., 2016). Nesse ínterim, tem-se proposto modelos de explicação de sua fisiopatologia e procurado o contínuo aperfeiçoamento de critérios de diagnóstico, tendo em vista o alto grau de incapacidade decorrente dessa enfermidade. Tendo como consequência uma maior predisposição para sintomas de depressão e ansiedade, levando o paciente a procurar assistência médica (JÚNIOR JOO e RAMOS JVC, 2019).

A etiologia e fisiopatologia da FM ainda permanecem desconhecidos. Os modelos propostos atualmente envolvem fatores externos (ambientais) e internos. Dentre os fatores ambientais destacam-se os papéis de traumas (físicos e psicológicos), estresse e traços interpessoais e estilo de vida. Por sua vez, os aspectos internos descritos incorrem em desequilíbrios nos mecanismos de sensibilização central e neurais periféricas (GALVEZ-SÁNCHEZ CM, et al., 2019).

Nesse contexto, a fisiopatologia da FM baseiam-se em: (1) aumento espontâneo da atividade de neurônios a nível da medula espinhal e tronco encefálico, em consequência da perda de inervação do sistema nervoso periférico; (2) hiperresponsividade de neurônios do sistema nervoso central devido a perda de nociceptores hiperativos da regulação inibitória do tronco cerebral, a nível da medula espinhal; (3) integração sensorial intersegmental e/ou suprassegmental bem desenvolvida; (4) alterações na rede de processamento sensorial talamocortical pelo envolvimento de neurotransmissores excitatórios e/ou a produção de citocinas inflamatórias (ALBRECHT PJ e RICE FL, 2016; THEOHARIDES TC, et al., 2015).

Além disso, no estudo de Ryabkova VA, et al. (2016) são enumeradas outras explicações para a FM. O trauma e a infecção apresentam como fatores precedentes ao componente autoimune da FM. Dentre os patógenos mais importantes se destaca os vírus da herpes simples, hepatite $\mathrm{C}$, epstein barr e a Borrelia burgdorferi. Além disso, pode estar relacionada a silicones mamários implantados, vacinas ou injeção de óleo mineral, induzidas por adjuvantes desses materiais. Outro componente do estudo foi a neuroinflamação com a análise de 92 proteínas, que evidenciou inflamação sistêmica crônica. A mais relevante foi a quimiocina CX3CL1, a qual faz conexão com a via de sinalização da dor neuropática. Também existe a explicação por ativação generalizada da micróglia, em maior quantidade nos lobos frontal e parietal, a qual foi associada a fadiga, dor e estresse. A neuropatia de pequenas fibras é outro mecanismo imunomediado que gera um gatilho periférico, repercutindo na sensibilização central, assim, potencializa a dor, bem como, o aumento do glutamato insular. 
Consequentemente, o quadro clínico de dor difusa crônica, fadiga e distúrbios do sono, os quais são os principais sintomas, coloca-se em evidência, juntamente com a possibilidade haver sintomas de depressão, ansiedade, desatenção, cefaleia, vertigens, parestesias, sintomas compatíveis com síndrome do intestino irritável ou com síndrome das pernas inquietas, entre diversos outros sintomas não relacionados ao aparelho locomotor (CLAUW DJ, 2014). Para tanto, Pernambuco AP, et al. (2017) obtiveram resultados em seu estudo clínico, os quais demonstram que pacientes com FM apresentam níveis significativamente altos de fadiga, distúrbios do sono, ansiedade e depressão, além de dores crônicas difusas, quando comparados a pacientes saudáveis.

Ao longo dos anos foram formulados diversos critérios para caracterizar o quadro clínico e confirmar o diagnóstico, entretanto, não existia unanimidade sobre esses pontos, gerando distorções diagnósticas. Nesse sentido, foi elaborado pelo Colégio Americano de Reumatologia (ACR) em 1990 os primeiros critérios, com valorização da dor difusa e busca de pontos dolorosos (HEYMANN RE, et al., 2017). Somente em 2010, o ACR criou novos critérios, adicionando alguns pontos, como a história de sintomas somáticos, o índice de dor generalizada (IDG) e a escala de gravidade dos sintomas (EGS, e eliminando a palpação dos pontos dolorosos. Posteriormente, houve diferentes modificações e ainda está em avaliação (WOLFE F, et al., 2010; WOLFE F, et al., 2018).

\section{Critérios diagnósticos}

Um primeiro ponto na avaliação da FM é a história clínica com destaque ao tempo de início, evolução, fatores desencadeantes, atenuadores e potencializadores, assim como, história familiar com parentes de primeiro grau e uso de medicações como estatinas, bifosfonatos e opioides. Outro ponto é o exame físico compatível com a idade, podem ter disestesia ao toque, rigidez miofascial ou mobilidade acentuada articular (HÄUSER W, et al, 2019). Para fazer o diagnóstico de FM, devem ser atendidos todos os seguintes critérios: dor generalizada em, pelo menos 4 de 5 locais; sintomas no nível similar no mínimo 3 meses; IDG $\geq 7$ e pontuação da EGS $\geq 5$ OU IDGI de 4-6 e pontuação EGS $\geq 9$; o diagnóstico de FM não descarta outras doenças e, independente de outros diagnósticos (WOLFE F, et al., 2016).

Heymann RE, et al. (2017) faz algumas recomendações para ajudar no diagnóstico da FM. (1) aplicar os critérios da ACR 1990 e 2010, para aumentar a acurácia; (2) avaliação da dor difusa, se suspeita; (3) inclusão dos pontos dolorosos quando associados a outros distúrbios funcionais; (4) considerar fadiga, distúrbios cognitivos e do sono. (5) não utilizar as modificações de 2011, que exclui sintomas somáticos; (6) não recomenda a termografia; (7) não recomenda a polissonografia; (8) não considerar FM como diagnóstico de exclusão; (9) recomenda mensurar os transtornos de humor. Dessa forma, inexistem marcadores laboratoriais e de imagem, além do quadro clínico ser variável, levando o diagnóstico da FM a ser baseado no julgamento clínico e variável com a experiência de cada médico.

Embora existam vários avanços no diagnóstico de FM, há muita incerteza, repercutindo em estresse, frustração e insatisfação do paciente. Quando se confirma a suspeita clínica de FM, muitas vezes, já aconteceram várias consultas, por vários especialistas, contribuindo tanto para questões sociais, quanto pessoais (HÄUSER W, et al, 2019). Em contraposição a esses desafios, em uma pesquisa da National Health Interview Survey (NHIS) com 8446 indivíduos evidenciou que $75 \%$ da população dos Estados Unidos com diagnóstico de FM eram falso-positivo (WALITT B, et al., 2016).

\section{Prevalência}

Di Tella M, et al. (2017) demonstraram em seu estudo que $77 \%$ de sua amostra relatou um alto nível de estresse emocional, incluindo 58\% relatando níveis relevantes de ansiedade e $63 \%$ de depressão. Outro estudo evidenciou que a fadiga e os distúrbios do sono foram associados a depressão elevada, mas não a ansiedade elevada, enquanto o pessimismo diante da dor, o medo do movimento e a intensidade da dor foram associados a depressão e ansiedade elevadas. Diante desse sofrimento psicológico, é frequentemente relatada a associação de Transtorno de Sintomas Somáticos em pacientes com fibromialgia (HADLANDSMYTH K, et al., 2017). 
Em relação à epidemiologia dos sintomas somáticos, no estudo de Axelsson E, et al. (2020), analisandose 140 pacientes com fibromialgia, 91 tinham pelo menos um sintoma somático, tendo como comorbidades: transtorno de ansiedade por doença (9 pacientes), depressão (10 pacientes), transtorno de ansiedade generalizada (6 pacientes). Ressalta-se, ainda, que 78 pacientes utilizavam medicamentos psicotrópicos.

Já no estudo de Hauser W, et al. (2020) no que diz respeito à prevalência da síndrome do desconforto corporal em indivíduos com fibromialgia, num total de 2531 pacientes, 80 apresentaram o subtipo cardiopulmonar, 77 o subtipo gastrointestinal e 142 o subtipo musculoesquelético.

Já o estudo de Heymann RE, et al. (2017), revela que, no Brasil, pacientes com fibromialgia têm risco aumentado de sintomas somáticos (67\%), depressão (55\%), síndrome do pânico (35\%) e agorafobia (30\%), bem como piores índices de dor, de qualidade do sono e de qualidade de vida.

\section{Repercussões na qualidade de vida}

A síndrome de fibromialgia é uma síndrome clínica que afeta diretamente a vida dos portadores da doença em virtude do conjunto sintomatológico relacionado a dores crônicas, e associado a episódios de ansiedade, depressão, alterações no padrão do sono, sendo complicações habituais nessa população, conforme abordado anteriormente. A detecção precoce da síndrome é um fator indicativo de melhor prognóstico, obtendo melhor qualidade de vida e funcionalidade a partir de um tratamento adequado. De acordo com Goulart R, et al. (2016) pacientes que apresentam uma pior condição de vida têm sintomas de exacerbação de depressão e ansiedade, resultando em aumento da concentração de comportamentos disfuncionais e incapacidade relacionada à doença como maior nível de abandono do tratamento.

O medo e a dor ao movimento causam danos psicológicos, considerando que indivíduos com fibromialgia têm sintomas generalizados associado ao sofrimento específico da dor, que podem impactar diretamente nos aspectos funcionais e no bem-estar, qualidade do sono, e interação com aspectos do cotidiano (HADLANDSMYTH K, et al., 2017).

A adesão ao tratamento e nível de comprometimento do paciente é a condição primordial e diretamente relacionada a fatores de melhora e qualidade de vida da síndrome de fibromialgia, porém devemos também considerar aspectos como idade, intensidade da dor, polifarmácia, qualidade da relação médico-paciente, circunstâncias socioeconômicas, a educação e outros parâmetros que afetam a vida humana. Nesse contexto, Oliveira Júnior JO e Ramos JVC (2019) mencionam que ao avaliarmos qualidade de vida envolverem conceitos como bem-estar físico, mental, psicológico e emocional, os relacionamentos sociais, como família e amigos, e também a saúde, a educação e outros parâmetros que afetam a vida humana.

A prática de exercícios físicos é um método favorável e benéfico para portadores de síndrome de fibromialgia, considerando que esse é hábito provocará liberação de hormônios como endorfina e serotonina, relacionados a sensação de prazer, modulação da dor, e sensação de bem-estar e equilíbrio. No entanto, para uma maior adesão dos pacientes, sugere-se exercícios de baixo impacto, viabilizando uma maior concentração, controle e precisão, respiração, como as práticas de pilates, melhorando o equilíbrio, flexibilidade, condicionamento físico, consciência corporal, postura, mobilidade articular, circulação sanguínea, encontrando um resultado eficaz e rápido (CORDEIRO BLB, et al., 2020).

A fibromialgia é uma doença persistente, sem cura, com tratamento exclusivamente sintomático, sendo necessário inserção de medidas farmacológicas e não farmacológicas, tornando importante o indivíduo aprender a lidar com situações da própria doença, adquirindo hábitos saudáveis, não relacionados somente ao tratamento medicamentoso, constituindo meios de convivência saudáveis, cuidados com o corpo, alimentação equilibrada, períodos para lazer, redes de apoio, avaliação multiprofissional, hábitos que propiciam satisfação e bem estar, melhorando e lidando melhor com aspectos da doença e estresse, melhorando o autocuidado em relação a sintomatologia, evitando desfechos de autoextermínio (OLIVEIRA JO e RAMOS JVC, 2019).

A escolha do enfrentamento do problema é a solução para aumentar a qualidade de vida e a resiliência em períodos de crise da síndrome de fibromialgia, além da assistência focada no apoio social e emocional, resultando em uma melhora do bem estar do indivíduo, visando a resiliência e menor vulnerabilidade em 
relação aos sintomas, adaptando-se mais facilmente, minimizando a ansiedade, depressão, a dor, controlando as emoções negativas, compartilhando experiências eficazes entre os grupos de pacientes, de acordo com gênero, idade e personalidade (BRAUNI A, et al., 2020).

\section{Tratamento}

Atualmente são utilizados diversos tipos de tratamento, tanto farmacológico quanto não farmacológico para pacientes com fibromialgia que possuem sintomas somáticos, o que garante uma melhor qualidade de vida para esses enfermos. Apesar de uma grande parcela dos pacientes apresentarem refratariedade com o manejo convencional, algumas alternativas de tratamento não farmacológico complementam as escolhas medicamentosas. Dentre essas alternativas, podemos citar a Terapia Cognitivo Comportamental (TCC), como importante aliada no tratamento da fibromialgia, pois pacientes que apresentam quadros álgicos crônicos tendem a desenvolver transtornos de humor e ansiedade, o que contribui para a piora do quadro clínico desses enfermos (HÄUSER W, et al., 2019). Além disso, o exercício físico, também, desenvolve um papel fundamental na busca do bem-estar destes doentes, pois, além de ser uma intervenção de baixo custo, diversos ensaios clínicos já constataram a eficácia da prática de atividades físicas na diminuição do quadro álgico em pacientes com dor crônica (OLIVEIRA L, et al., 2017).

O biofeedback, chamado de terapia psicofisiológica, também, trata-se de outra intervenção não farmacológica que propicia aos portadores de fibromialgia a tomarem consciência de práticas que maximizam as manifestações clínicas da doença. Por meio de sensores elétricos que repassam informações sobre o corpo, essas pessoas poderão implementar mudanças sutis em seu comportamento, possibilitando uma melhor qualidade de vida, devido a diminuição do quadro álgico. Ademais, outras possibilidades não farmacológicas se apresentam como importantes coadjuvantes na busca de melhorar o cotidiano dos portadores dessa enfermidade, como a hipnose, que auxilia no desenvolvimento de uma consciência plena e focalizada, diminuindo os distratores ambientais e ajudando na diminuição dos principais sintomas somáticos. Além destes, a hidroterapia, a massoterapia, o iogo e outras atividades relaxantes, contribuem para o tratamento da doença, pois diminuem o tônus muscular e auxiliam em um melhor repouso dos pacientes e na melhoria do bem-estar desses indivíduos (HÄUSER W, et al, 2019).

Dentre as alternativas farmacológicas, disponibilizamos dos antidepressivos, tanto os Inibidores Seletivos da Recaptação de Serotonina (ISRS), quanto os antidepressivos tricíclicos, no qual podemos destacar a amitriptilina, que além de aumentar a disponibilidade da serotonina e noradrenalina nas fendas sinápticas, têm uma ação analgésica central, o que contribui para o alívio da dor e para a melhora da fadiga e do sono. Outros fármacos que poderão ser utilizados são anticonvulsivantes, que além do alívio das crises álgicas, minimizam outros sintomas, como a fadiga muscular. Analgésicos e anti-inflamatórios não esteroidais, também, são outras opções para o tratamento das manifestações clínicas da fibromialgia (JUNIOR JOO e ALMEIDA MB, 2018).

\section{CONSIDERAÇÕES FINAIS}

Diante dos estudos apresentados sobre a síndrome da fibromialgia, foi elucidado que há uma prevalência de sintomas somáticos nos pacientes diagnosticados, sendo as manifestações mais relatadas: dores crônicas, ansiedade e depressão. Esta síndrome afeta diretamente a vida de pacientes portadores dessa patologia e seu diagnóstico é relativo, pois irá depender da análise do quadro clínico e do entendimento do médico escolhido, uma vez que para reconhecê-la não existem métricas definidas disponíveis até o momento. Sendo assim, é relevante que continuem sendo realizados estudos científicos a respeito do assunto, a fim de propor formas para mensurar o grau de intensidade e dor causado por essa doença e tratamento precoce específico e, por fim, tais descobertas irão ajudar na melhoria na qualidade de vida, bem-estar físico e mental das pessoas que a possuem.

\section{REFERÊNCIAS}

1. ALBRECHT PJ, RICE FL. Fibromyalgia syndrome pathology and environmental influences on afflictions with medically unexplained symptoms. Reviews on environmental health, 2016; 31(2): 281-294. 
2. AXELSSON E, et al. Symptom Preoccupation in Fibromyalgia: prevalence and correlates of somatic symptom disorder in a self-recruited sample. Psychosomatics, 2020; 61(3): 268-276.

3. BIRTANE M, et al. The evaluation of quality of life in fibromyalgia syndrome: a comparison with rheumatoid arthritis by using SF-36 Health Survey. Clinical rheumatology, 2007; 26(5): 679-684.

4. BRAUN A, et al. Clustering fibromyalgia patients: A combination of psychosocial and somatic factors leads to resilient coping in a subgroup of fibromyalgia patients. Plos one, 2020; 15(12): e0243806.

5. CLAUW DJ. Fibromyalgia: A Clinical Review. Journal of the American Medical Association, 2014; 311(15): 15471555.

6. CORDEIRO BLB, et al. Influence of the Pilates method on quality of life and pain of individuals with fibromyalgia: integrative review. Sociedade brasileira para estudo da dor, 2020; 3(3): 258-62

7. GALVEZ-SÁNCHEZ CM, et al. Psychological impact of fibromyalgia: current perspectives. Journal of Psychology Research and Behavior Management, 2019; 12: 117-127.

8. GOULART R, et al. Aspectos psicológicos da síndrome da fibromialgia juvenil: revisão de literatura. Revista Brasileira de Reumatologia, 2016; 56(1): 69-74.

9. HADLANDSMYTH K, et al. Somatic symptom presentations in women with fibromyalgia are differentially associated with elevated depression and anxiety. Journal of health psychology, 2017; 25(6): 819-829.

10. HARRINSON J, et al. Revising the ICD: explaining the WHO approach. The Lancet, 2016; 388(10059): $2476-2477$.

11. HÄUSER W, et al. Construct validity and clinical utility of current research criteria of DSM-5 somatic symptom disorder diagnosis in patients with fibromyalgia syndrome. Journal of psychosomatic research, 2015; 78(6): 1-16.

12. HÄUSER W, et al. Fibromyalgia syndrome: under-, over- and misdiagnosis. Clinical and Experimental Rheumatology, 2019; 37(1): S90-S97.

13. HEYMANN RE, et al. Novas diretrizes para o diagnóstico da fibromialgia. Sociedade Brasileira de Reumatologia, 2017; 57(S2): S467- S476

14. JÚNIOR JOO, RAMOS JVC. Adherence to fibromyalgia treatment: challenges and impact on the quality of life. Revista Brazilian Journal of Pain, 2019; 2(1): 81-87.

15. MARQUES AP, et al. Prevalence of fibromyalgia: literature review update. Revista Brasileira de Reumatologia. 2017; 57(4): 356-363.

16. MCBETH J, et al. Features of somatization predict the onset of chronic widespread pain: results of a large populationbased study. Arthritis \& Rheumatism, 2001. 44(4): 940-946.

17. OLIVEIRA JO, RAMOS JVC. Adherence to fibromyalgia treatment: challenges and impact on the quality of life. Sociedade brasileira para estudo da dor, 2019; 2(1): 81-7.

18. JUNIOR JOO; ALMEIDA MB. The current treatment of fibromyalgia. BrJP, 2018; 1(3): 255-262.

19. OLIVEIRA $L$, et al. Efeito do exercício físico supervisionado na flexibilidade de pacientes com fibromialgia. Rev. dor, São Paulo, 2017; 18(2): 145-149.

20. PERNAMBUCO AP, et al. Clinical profile of patients with fibromyalgia syndrome. Revista Fisioterapia em Movimento. 2017; 30(2): 287-296.

21. RYABKOVA VA, et al. Neuroimmunology: What Role for Autoimmunity, Neuroinflammation, and Small Fiber Neuropathy in Fibromyalgia, Chronic Fatigue Syndrome, and Adverse Events after Human Papillomavirus Vaccination?. International Journal of Molecular Sciences, 2019; 20(20): 5164.

22. SOUZA JB, PERISSINOTTI DM. A prevalência da fibromialgia no Brasil - estudo de base populacional com dados secundários da pesquisa de prevalência de dor crônica brasileira. BrJP, 2018; 1(4): 345-348.

23. THEOHARIDES TC, et al. Fibromyalgia syndrome in need of effective treatments. Journal of Pharmacology and Experimental Therapeutics, 2015; 355(2): 255-263.

24. VLAEYEN JWS, LINTON SJ. Fear-avoidance model of chronic musculoskeletal pain: 12 years on. Pain, 2012; 153(6): 1144-1147.

25. WALITT B, el al. Three-Quarters of Persons in the US Population Reporting a Clinical Diagnosis of Fibromyalgia Do Not Satisfy Fibromyalgia Criteria: The 2012 National Health Interview Survey. PLOS ONE, 2016; 11(6): e0157235

26. WOLFE $F$, et al. Fibromyalgia diagnosis and biased assessment: Sex, prevalence and bias. Revista PLoS ONE, 2018; 13(9): e0203755.

27. WOLFE F, et al. 2016 Revisions to the $2010 / 2011$ fibromyalgia diagnostic criteria. Seminars in Arthritis and Rheumatism, 2016; 46(3): 319-329.

28. WOLFE, et al. The American College of Rheumatology Preliminary Diagnostic Criteria for Fibromyalgia and Measurement of Symptom Severity. Wiley Online Library, 2010; 62(5): 600-610. 